\title{
CALIFICACIÓN METADISCURSIVA Y METÁFORA EN CONVERSACIONES ENTRE ADOLESCENTES
}

\author{
Lucía BREGANT \\ CONICET / Universidad de Buenos Aires
}

\section{RESUMEN}

Este trabajo tiene como objetivo estudiar los procedimientos lingüísticotextuales mediante los cuales sujetos adolescentes reformulan expresiones metafóricas en un contexto conversacional. Me propongo ahondar en la naturaleza de los recursos utilizados en la reformulación y analizar, específicamente, los procedimientos de calificación metadiscursiva (Gülich y Kotschi 1995; Ciapuscio 2007a y 2007b), mediante los cuales los hablantes comentan y/o evalúan las expresiones metafóricas; en el trabajo se analiza su presencia, distribución y variación en intercambios entre sujetos adolescentes a los que se les ha solicitado que reformulen expresiones metafóricas en el contexto de una conversación cara a cara. Intento dilucidar en qué medida el tipo y la frecuencia de los recursos de calificación metadiscursiva que acompañan y señalan los procedimientos de reformulación se correlacionan con el grado de convencionalidad de las expresiones metafóricas reformuladas, y, subsidiariamente, aportar a las investigaciones sobre la metáfora en corpus textual, específicamente, a la pregunta sobre el carácter convencional vs. novedoso de la metáfora (Hanks 2004, 2006).

PALABRAS CLAVE: adolescentes, calificación metadiscursiva, convencionalidad, metáfora, procedimientos de formulación, reformulación.

\section{AbSTRACT}

This paper studies the linguistic and textual procedures through which adolescent subjects reformulate metaphorical expressions in a conversational context. My purpose is to examine the nature of the resources used in said reformulation, and to specifically analyze the procedures of metadiscursive qualification (Gülich and Kotschi 1995; Ciapuscio 2007a, 2007b) through which speakers comment and/or evaluate metaphorical expressions: the study reviews their presence, distribution and variation in exchanges among adolescent subjects who have been asked to reformulate metaphorical expressions in the context of a face-to-face conversation. My aim is to elucidate the extent to which the type and frequency of metadiscursive qualification accompanying and signaling reformulation processes are correlated with the degree of conventionality of the reformulated metaphori- 
cal expressions and, additionally, to contribute to the research on metaphor in a text corpus, especially, to the question regarding the conventional vs. innovative character of metaphors (Hanks 2004, 2006).

KEYWORDS: adolescents, conventionality, formulation procedures, metadiscursive qualification, metaphor, reformulation.

\section{INTRODUCCIÓN}

El presente trabajo forma parte de una investigación que tiene como objetivo estudiar los procedimientos textuales mediante los cuales sujetos adolescentes reformulan expresiones metafóricas en un contexto conversacional $^{1}$. Los resultados de un trabajo previo sobre los mismos datos mostraron que los sujetos tienden a usar diferentes estrategias discursivas en la reformulación de metáforas de acuerdo con el grado de convencionalidad que estas presenten (Bregant 2015). En esta oportunidad, intentaré ahondar en la naturaleza de los recursos utilizados en la reformulación y analizar, específicamente, los procedimientos de calificación metadiscursiva, mediante los cuales los hablantes comentan y evalúan el discurso en desarrollo (Kotschi 1986; Gülich y Kotschi 1995; Ciapuscio 2007a y 2007b). Resulta de interés indagar en la forma en que estos procedimientos textuales se usan en contextos orales y espontáneos, en los que prima la negociación de significados.

Las preguntas iniciales son: a) ¿qué recursos de calificación metadiscursiva se seleccionan para acompañar procedimientos de reformulación de metáforas de diferentes grados de convencionalidad?; b) ¿pueden establecerse diferencias en la cantidad y el tipo de recursos que emplean los hablantes según el tipo de metáforas que se reformulen, esto es, según el mayor o menor grado de convencionalidad?

\section{MARCO TEÓRICO}

Dado que en este trabajo se pretende articular dos fenómenos lingüísticos de diferente naturaleza, la metáfora y los procedimientos discursivos

${ }^{1}$ El título del proyecto es «Procedimientos y marcadores de producción discursiva en textos de adolescentes rioplatenses». Se trata de mi tesis de doctorado en curso, que se encuentra financiada por una Beca Doctoral de CONICET, bajo la dirección de la Dra. Guiomar Ciapuscio. 
de calificación y de reformulación, el marco teórico de referencia es consecuentemente heteróclito: por un lado, la teoría conceptual de la metáfora y, por el otro, la teoría de la producción discursiva.

Los estudios sobre metáfora de orientación cognitiva (Lakoff y Johnson 1980, 1999; Lakoff 1993; Kövecses 2002) han resaltado la omnipresencia de este fenómeno en textos cotidianos y su importancia para el pensamiento y la conceptualización del entorno. Se trata de una proyección entre un dominio fuente y un dominio meta que puede expresarse lingüísticamente de diferentes maneras. Sin embargo, las metáforas no sólo constituyen un procedimiento cognitivo, sino que son también recursos comunicativos de naturaleza interaccional (Ciapuscio 2005). En consecuencia, diferentes autores han señalado la importancia del estudio de la metáfora en corpus lingüísticos que proporcionen datos empíricos sobre su realización discursiva (Cameron 2003; Cameron y Deignan 2003; Deignan 2008; Steen 2007; Hanks 2004, 2006, 2013, entre otros). Con este propósito, se han realizado diversos trabajos en lengua española, especialmente abocados al estudio de la metáfora en un ámbito específico de la lengua, como es el caso del discurso político (Hernández Miranda 2015 y Duarte 2013, entre otros), el discurso médico y científico (Ciapuscio 2005, 2016, entre otros), el discurso periodístico (Olza Moreno 2008, entre otros) y el discurso publicitario (Ribas y Todolí 2008, entre otros). En lo que respecta a la conversación, Briz (1998) destaca la rentabilidad del uso de expresiones metafóricas en el habla coloquial. Para determinar las principales características formales y funcionales de la metáfora en la conversación coloquial, Fernández Colomer (2003) realiza un estudio a partir del corpus del grupo Val.Es.Co (Briz et al. 1995). Concluye que las metáforas lexicalizadas son las predominantes y que cumplen las funciones de orientar la posición argumentativa de los hablantes y vehiculizar la expresividad y la intensificación.

La diferenciación entre expresiones lingüísticas metafóricas y literales es una cuestión de grado, así como lo es la convencionalidad de una metáfora. De acuerdo con la Teoría de las normas y las explotaciones (Hanks 2004, 2013), existen patrones de comportamientos lingüísticos asociados a cada palabra que constituyen su norma; cualquier uso genuino que no se ajuste a esta norma es una explotación de la misma. En principio, las metáforas serían casos de explotaciones: «reconstruir el puente» constituiría la norma, mientras que «reconstruir su vida» sería una explotación de esta norma. Sin embargo, cuando una expresión metafórica está lo suficientemente convencionalizada (esto es, cuando es lo suficientemente recurrente en el uso), puede convertirse en norma: por ejemplo, «corazón sin amor». La frecuencia de uso sería, entonces, un criterio para determinar el grado de convencionalidad de una expresión metafórica. Por otro lado, diacrónicamente, las metáforas presentan una evolución que puede llevarlas a la 
lexicalización, que tiene como consecuencia su registro lexicográfico. Finalmente, desde el punto de vista del procesamiento, según Lakoff (1993), las metáforas convencionales, que se procesan automáticamente, son la base para las metáforas nuevas, que requieren de un mayor esfuerzo en el procesamiento. En cualquier caso, frecuencia de uso, lexicalización y dificultad en el procesamiento son criterios para determinar el grado de convencionalidad que se relacionan fuertemente entre sí y deben concebirse en términos de un continuum.

En este trabajo se analiza la incidencia de los diferentes grados de convencionalidad de una expresión metafórica en los procedimientos de reformulación, focalizando en los recursos de calificación empleados en intercambios lingüísticos dialogales. Para esto, se toma como segundo marco teórico de referencia la teoría de la producción discursiva. Según esta teoría (Antos 1982; Gülich y Kotschi 1987, 1995; Gülich 2006; Ciapuscio 2007a, 2007b), producir un texto es un trabajo progresivo de resolución de problemas que los interlocutores llevan a cabo en colaboración. Para realizar esta tarea, los hablantes contamos con una serie de «etnométodos»: métodos que los miembros de una sociedad tenemos a nuestra disposición para resolver los problemas prácticos de nuestras conversaciones. Cuando estos obstáculos surgen, el trabajo de producción discursiva del hablante deja huellas en sus expresiones lingüísticas, que entonces son accesibles para el análisis. Gülich y Kotschi (1995) distinguen tres conjuntos de huellas que funcionan como base para identificar tres tipos de procedimientos discursivos: los procedimientos de verbalización, los de tratamiento y los de calificación. Los primeros dan cuenta del hecho mismo de «poner en palabras» contenidos; se trata mayormente de fenómenos de parole o performance. Sus huellas características son los marcadores paramorfemáticos, como los falsos comienzos, las repeticiones, los alargamientos o las pausas («si pero, $e: h$ le p/ lo que le po:nen esas co:sas,. si,» ${ }^{2}$ ). Los procedimientos de tratamiento son procedimientos mediante los cuales los hablantes se refieren a un segmento precedente por medio de una nueva expresión, que de algún modo cambia, modifica, reformula o expande la expresión previa (véase el ejemplo 1 abajo). Esta categoría incluye las reformulaciones y los procedimientos no reformulativos, como las generalizaciones y las ejemplificaciones. Sus huellas características son diferentes tipos de marcadores conectores, que suelen ser verbales, aunque pueden no serlo (también pueden ser marcadores de tratamiento la entonación, las pausas o algunos gestos). Por último, los procedimientos de calificación se utilizan para evaluar o comentar, de manera metadiscursiva expresiones que forman parte del discurso en desarrollo y haciéndolo señalan ciertos problemas de verbalización o de

${ }^{2}$ Las convenciones de transcripción se detallan al final del trabajo. 
comunicación. Sus huellas características son diferentes tipos de marcadores que funcionan como operadores y que suelen incluir elementos metalingüísticos (véanse los ejemplos 2, 3 y 4 abajo). Así, el procedimiento metadiscursivo por el que se da cuenta de los problemas en la producción discursiva recurre, en su formulación, a elementos metalingüísticos, que señalan expresiones lingüísticas del discurso.

En este trabajo se atenderá especialmente a los procedimientos de calificación y su relación con los de reformulación. El estudio de esta relación responde a resultados obtenidos por otros autores (Kotschi 1986; Gülich y Kotschi 1995; Ciapuscio 2007a y 2007b), en el sentido de que los procedimientos de calificación metadiscursiva aparecen habitualmente ligados a una (o varias) reformulación(es), de manera que los hablantes a la vez que califican expresiones del discurso simultáneamente las reelaboran. Asimismo, se trata de procedimientos semejantes en tanto se encuentran en un nivel metadiscursivo: tanto la reformulación como la calificación interrumpen el discurso para aportar información sobre los constituyentes del discurso mismo. Sin embargo, presentan estructuras y marcadores diferentes que justifican su clasificación en diferentes tipos, si bien los autores destacan que no se trata de una categorización rígida con líneas divisorias claras.

Desde este punto de vista, la reformulación se caracteriza por presentar una estructura prototípica tripartita: una expresión de referencia (explícita o implícita); un marcador verbal o no verbal y una expresión de tratamiento sugerida como alternativa (ejemplo 1):

Expresión de Referencia (ER) ${ }^{3}+$ Marcador Discursivo (MD) + Expresión de Tratamiento (Reformulación) (ET)

(1) es igual que todas las personas (ER), o sea: (MD) no cambió: (ET) no sabe más que otras personas

En el marco de la teoría de la relevancia, Blakemore $(1993,2002)$ realiza una diferencia entre la reformulación planificada y la no planificada. Es sólo en el último caso, característico de la oralidad, que la reformulación responde al reconocimiento por parte del hablante de que su enunciado no ha sido exitoso comunicativamente. Por esto, los marcadores utilizados para reformular en los textos escritos y en los orales muchas veces difieren (Figueras 2001).

${ }^{3}$ ER y ET pueden ser segmentos textuales del mismo hablante o de hablantes distintos. En el primer caso, se trata de autorreformulación; en el segundo, de heterorreformulación. Asimismo, de acuerdo con quién inicie la operación de reformular, puede distinguirse entre reformulación autoiniciada y reformulación heteroiniciada (Schegloff, Jefferson y Sacks 1977; Gülich y Kotschi 1987). 
Desde el punto de vista del significado, la reformulación presenta, al mismo tiempo, cierto grado de equivalencia y cierto grado de distancia entre la ER y la ET. A partir de este presupuesto, muchos estudios sobre reformulación tradicionalmente parten de la distinción entre reformulación parafrástica y no parafrástica ${ }^{4}$ de acuerdo con el grado de semejanza que se adjudica entre el elemento reformulador y el reformulado. Otros autores (Pons 2013; Garcés Gómez 2008), sin embargo, señalan que este tipo de clasificación resulta inconsistente, en tanto la noción de distancia no termina de definirse, especialmente teniendo en cuenta que desde el momento en que hay una reformulación el hablante está estableciendo cierta distancia con la formulación inicial (Pons 2013: 155).

En cuanto a los procedimientos de calificación metadiscursiva, presentan una estructura binaria: una expresión calificada -el enunciado fuente (EF)-, y una expresión calificadora (EC). El orden secuencial es variable, si bien la EC suele ser posterior. Kotschi (1986) y luego Gülich y Kotschi (1995), sobre la base de un amplio corpus de intercambios orales en lengua francesa, diferencian tres tipos de calificaciones metadiscursivas:

a) Evaluaciones. Consisten en dos elementos: primero, una expresión anafórica con la estructura $(\mathrm{X}) \mathrm{Y}(\mathrm{Z})$, siendo X la expresión tratada, Y un elemento anafórico ${ }^{5}$ y Z una expresión metalingüística; segundo, una expresión evaluativa, que típicamente contiene una expresión predicadora (ejemplo 2).

(EF) (elemento anafórico(EA)) (expresión metalingüística (EM)) + expresión evaluativa (EE)

(2) es un término (EM) vulgar (EE)

b) Comentarios. Contienen expresiones calificadoras que tienen por lo menos un elemento metalingüístico (EM) como su constituyente principal. Además, pueden contener un elemento anafórico (EA) o catafórico (ejemplo 3).

(elemento anafórico) + expresión metalingüística

(3) no sé cómo explicar(EM) lo(EA)

${ }^{4}$ Algunas de las propuestas de clasificación que realizan esta distinción son Gülich y Kotschi (1987, 1995); Roulet (1987); Rossari (1994); Fuentes (1993); Bach (2000) (cfr. Pons, 2013).

${ }_{5}$ Para el español, Ciapuscio (2007a y 2007b) observa que la expresión anafórica es poco frecuente, aunque sí lo es la remisión al enunciado fuente mediante algún elemento metalingüístico, seguido de la expresión evaluativa. 
c) Atenuadores (hedges). Son inserciones (del tipo «una especie de», «en cierto modo») que muestran la vaguedad o indeterminación del enunciado fuente (ejemplo 4).

(4) SÍ, que se enfermó su alma,. sería como se... como le rompió el alma,.. algo asi

Desde el punto de vista de su estructura los atenuadores no se caracterizan por la inclusión de elementos metalingüísticos. Tienen un alcance más local que los otros miembros de la clase y constituyen una categoría heterogénea. Por estos motivos, en Ciapuscio (2007a: 65) se argumenta que los atenuadores no se encuentran en un nivel semejante a las evaluaciones y los comentarios metadiscursivos. En efecto, la atenuación involucra elementos de diferentes niveles (además del marcador como pueden apreciarse en el ejemplo anterior el tiempo verbal, la vacilación y el algo así). Siguiendo esta línea, en este trabajo se relevaron únicamente las evaluaciones y los comentarios, mientras que los atenuadores se tuvieron en cuenta únicamente en aquellas secuencias textuales que presentaran los otros procedimientos, con los cuales, habitualmente, se combinan. Asimismo, la distinción entre comentarios y evaluaciones fue concebida en términos de un continuum caracterizado por los polos [-calificación] y [+calificación] ${ }^{6}$.

\section{MATERIALES y MÉTODOS}

Para la conformación del corpus ${ }^{7}$ que analizo en este trabajo se adaptó el método Goal-Directed Interactive Think Aloud (GITA), desarrollado por Cameron (2003: 153) para el estudio de la comprensión de metáforas en niños. Se trata de una técnica adaptada del Think Aloud, en la que se les pide a los individuos que reporten sus pensamientos a medida que van leyendo un texto. A diferencia de esta última, GITA proporciona una guía para la realización de la tarea de lectura, que funciona reduciendo la complejidad de los recursos metacognitivos puestos en juego y asemejando la realización de la tarea planteada por el investigador a la actividad cotidiana de comprensión textual que los sujetos llevan a cabo en el ámbito escolar.

\footnotetext{
${ }^{6}$ Según ha observado Ciapuscio (2007a: 72), es conveniente subdividir la clase de los comentarios en denominativos y valorativos. Los primeros presentan el menor grado de calificación y se encuentran más estandarizados e integrados al discurso; los segundos, tienen un grado mayor de calificación. En el polo contrario del continuum se encontrarían las evaluaciones, que se caracterizan por un alto grado de calificación y un bajo grado de estandarización e integración.

${ }^{7}$ El presente corpus constituye una muestra del corpus recogido en el trabajo de tesis doctoral.
} 
Participaron 18 sujetos, 6 varones y 12 mujeres, de entre 16 y 18 años de edad, divididos en 5 grupos $^{8}$. Se distribuyó una selección de 5 a 7 canciones diferentes en cada grupo, con las siguientes indicaciones: en primer lugar, se les solicitó que hablaran de las canciones en general, que dijeran de qué se trataban y las explicaran; en segundo lugar, que intentaran identificar el género musical de procedencia de cada canción y justificaran el porqué de tal clasificación; en tercer lugar, que dieran su opinión personal de cada canción. Si bien en la resolución de estas tres tareas los hablantes suelen realizar actividades de reformulación, se planificó la cuarta consigna para asegurar la elicitación de reformulaciones. En esta consigna se les solicitó a los sujetos que explicaran el significado de las palabras y frases, mayormente metafóricas, destacadas en los textos. Los intercambios orales producto de la realización de las tareas suman 230 minutos de conversación.

El primer paso del análisis consistió en el relevamiento de las metáforas del corpus que hubieran suscitado un tratamiento reformulativo. Para determinar el grado de convencionalidad de las metáforas se recurrió a la búsqueda lexicográfica ${ }^{9}$ y a la consulta de bibliografía que documenta metáforas conceptuales (Lakoff y Johnson 1980, 1999; Lakoff, Espenson y Schwartz 1991; Lakoff 1993; Kövecses 2002). El motivo de este doble criterio para la determinación de la convencionalidad radica en el carácter a la vez lingüístico y conceptual de las metáforas. En primer lugar, en tanto expresiones lingüísticas, muchas veces las metáforas altamente convencionales se lexicalizan, de manera que pueden encontrarse en los diccionarios del español. Ese es el caso, por ejemplo, de mamado con el sentido de 'borracho' (cfr. infra: ejemplo 6). En segundo lugar, en tanto procedimiento cognitivo, las metáforas conceptuales indican un conjunto de proyecciones entre el dominio fuente y el dominio meta que pueden manifestarse de muchas formas, entre ellas el lenguaje (Soriano 2012). Para una misma metáfora conceptual subyacente, existen diferentes expresiones lingüísticas posibles, muchas de las cuales no están lexicalizadas. Así, como se verá en el ejemplo 7, para la metáfora conceptual MALO ES ABAJO encontramos lexicalizado el sentido metafórico de caer, pero no en todos los diccionarios el de tirado. Por este motivo, y teniendo en cuenta que el grado de convencionalidad se entiende en términos de continuum, se utilizaron estos dos criterios como complementarios, con predominancia del criterio lexicográfico, en tanto se trata de un trabajo lingüístico. Se clasificaron como más convencionales aquellas expresiones metafóricas que se encuentran

${ }^{8}$ La experiencia se realizó en dos escuelas públicas céntricas de la zona Oeste del área metropolitana de la Provincia de Buenos Aires, de niveles académico y sociocultural similares.

${ }^{9}$ Las obras lexicográficas consultadas para tal fin fueron: Battaner (2003), Plager (2008), Real Academia Española (2015) y Conde (2011). 
registradas en los diccionarios y/o documentadas en la bibliografía especializada sobre el tema, como menos convencionales aquellas que no cumplieran con estos criterios o los cumplieran a medias, y como metáforas nuevas aquellas que no cumplieran con ninguno. Se establecieron, a modo de referencia, tres grados en el continuum de convencionalidad de una metáfora: grado 1 para las metáforas más convencionalizadas, grado 2 para las menos convencionalizadas y grado 3 para las nuevas.

El análisis de los intercambios fue de tipo cualitativo y se realizó siguiendo los principios metodológicos de la teoría de la producción discursiva. Se atendió a la dimensión interactiva, ya que la producción textual es entendida en este marco como un trabajo colaborativo. Asimismo, se priorizó el análisis secuencial, en tanto el discurso es considerado un proceso que se desarrolla progresivamente, en lugar de un producto acabado (Gülich 2006). Los fenómenos lingüísticos observables, relevados y analizados, funcionan como indicadores de los procedimientos subyacentes.

Dado que se estudió un corpus textual extenso, a continuación presentaré solamente algunos ejemplos paradigmáticos que permitan mostrar las regularidades encontradas en el análisis.

\section{ANÁlisis}

\subsection{Grado 1: Metáforas convencionales}

El primer conjunto de intercambios analizados está conformado por aquellas reformulaciones que surgieron a partir de expresiones metafóricas con un alto grado de convencionalidad. La tendencia que observé, en estos casos, es a que la reformulación se produzca con un grado bajo de colaboración entre los hablantes: la tarea de reformular queda a cargo de uno solo de los sujetos que participan en el intercambio, mientras que sus interlocutores se limitan a asentir, pero sin proponer reformulaciones alternativas o complementar la reformulación inicial. Las secuencias de tratamiento suelen ser breves y hay escasa o nula presencia de calificación metadiscursiva, como sucede en el siguiente ejemplo $(5)^{10}$ :

(5) Tengo amigos nuevos para querer. / Escucho sus consejos para aprender. / Siempre están a mi lado, cuidándome. / No quieren verme tirado y no lo haré.

${ }^{10}$ A continuación, y en todos los ejemplos que siguen, transcribo en primer lugar el fragmento de la canción sobre la que los participantes hablan y de la que se extrae la expresión de referencia (ER), que se destaca en cursiva, y, a continuación, el intercambio conversacional. Asimismo, uso la cursiva para destacar las huellas de calificación metadiscursiva presentes en los intercambios. 


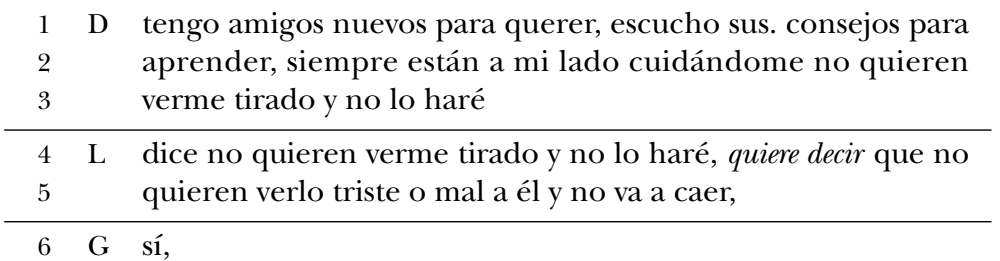

La expresión de referencia contiene una metáfora convencionalizada, en tanto puede identificarse con las metáforas conceptuales FELIZ ES ARRIBA / TRISTE ES ABAJO y MALO ES ABAJO (Lakoff y Johnson 1980; Lakoff, Espenson y Schwartz 1991), y resulta fácilmente interpretable para los hablantes. Sin embargo, como expresión lingüística no presenta un grado de lexicalización tan alto, ya que el DIEA y el DUEAE no recogen este sentido ${ }^{11}$. El pasaje entre dominios fuente y meta es fluido: el hablante a cargo de la reformulación (L) remite al dominio meta con «triste» (y también con «mal»), que se presentan como expresiones equivalentes a la que se observa en la canción, pero en seguida regresa al dominio fuente con la metáfora «caer». En este caso, se trata de una expresión metafórica distinta que tiene como base la misma metáfora conceptual subyacente que la ER. Esta metáfora presenta un grado de lexicalización mayor, ya que es recogida en su sentido metafórico por todos los diccionarios consultados. No se encuentran calificadores ni evaluadores en esta secuencia, si bien la dimensión metalingüística está presente en el marcador de reformulación quiere decir en la línea 4. Los otros participantes de la conversación se limitan a la lectura del fragmento textual a reformular (D, en las líneas 1 a 3) y a acordar con la reformulación propuesta ( $\mathrm{G}$, en la línea 6 ), pero no llevan a cabo el trabajo reformulativo propiamente dicho.

En el ejemplo 6, en el que se reformula la expresión metafórica mamados, que también presenta un alto grado de convencionalidad, ya que está lexicalizada ${ }^{12}$, tampoco se observan evaluaciones o comentarios metadiscursivos.

(6) ¿A dónde están los mamados? / Ahí, ahí.

$1 \mathrm{~S}$ «mamados». o sea que están re en pedo

2 L que está re borracho

3 M que estás alcoholizada

\footnotetext{
${ }^{11}$ Sí lo hacen el DRAE, 'Que está mal de salud o de ánimo' y Conde (2011): ‘sentirse decaído'.

${ }^{12}$ Los diccionarios de lengua general consultados registran mamarse como 'emborracharse'.
} 
En este intercambio, S presenta la expresión metafórica de referencia y la reformula inmediatamente mediante otra expresión figurada convencional y lexicalizada: está en re pedo. El marcador de reformulación o sea funciona como huella clara de este procedimiento de reformulación. A diferencia de lo que sucedía en el ejemplo anterior (5), en este caso no hay remisiones al dominio fuente: las reformulaciones sucesivas se quedan en el dominio meta. Las propuestas de reformulación que realizan L y M aparecen, antes que corrigiendo, reforzando la reformulación original. No se encuentran marcadas por ningún elemento metadiscursivo y son expresiones sinónimas, que abandonan el campo de lo metafórico. La reformulación se realiza colaborativamente: todos los participantes realizan su propuesta de reformulación, pero sin esfuerzo aparente, en tanto no hay discusiones explícitas, metadiscursivas, en torno al trabajo de reformulación y la selección léxica que conlleva.

\subsection{Grado 2: Metáforas menos convencionales}

Ubico en este conjunto al sector medio de la escala de convencionalidad, aunque se trata de un corte arbitrario realizado operativamente para dar cuenta de un fenómeno gradual. En principio, son expresiones metafóricas no lexicalizadas, aunque pueden estar en vías de lexicalizarse, y para las que es difícil reconocer una metáfora conceptual subyacente que se encuentre registrada en la bibliografía sobre el tema. Sin embargo, como veremos a continuación, son varios los factores que afectan el grado de convencionalidad que puede atribuírsele a una expresión metafórica, dado que también entra en juego el conocimiento que tengan de ella los sujetos que la formulan o reformulan. Las reformulaciones de este grupo intermedio fueron las que presentaron, en este corpus, mayor cantidad y variedad de calificaciones metadiscursivas. Estas pueden señalar la dificultad, pero, en ocasiones, también la facilidad en la tarea de reformulación:

(7) Jubilados de la corrupción / que te mojan la oreja. / Ni te piden perdón. / juegan con la inocencia de tu generación.

\begin{tabular}{rrl} 
1 & C & donde dice, jubilados de la corrupción que te mojan la oreja,. \\
\hline 2 & D & corrupción. jubilados,. \\
\hline 3 & B & corrup/ eso: no entendí,. que te mojan la oreja, \\
\hline 4 & M & sí, \\
\hline 5 & B & y te piden perdó:n’ \\
\hline 6 & D & que le bajan el sueldo a los jubilados' no, \\
7 & B & a:h mirá \\
\hline
\end{tabular}


$8 \quad$ B ni te piden perdón

\begin{tabular}{rll}
\hline 9 & M & mojan la oreja sería una metáfora, \\
\hline 10 & B & sí, por eso, \\
\hline 11 & C & sí: \\
\hline 12 & B & y te piden perdón \\
13 & C & y te piden perdón <al unísono $>$ \\
\hline 14 & C & juegan con la inocencia de tu generación, \\
\hline 15 & D & y s / te mienten, yo qué sé, \\
\hline 16 & C & te mienten, \\
\hline 17 & M & o sea te mojan la oreja: a:h \\
\hline 18 & C & claro... \\
\hline 19 & B & te joden,. podemos decir, no’ \\
\hline 20 & M & sí
\end{tabular}

En el ejemplo 7 se reformula la frase hecha mojar la oreja, a la que, en principio, podría atribuírsele un grado de convencionalidad alto, en tanto se encuentra registrada en el $D I E A^{13}$. Sin embargo, para los sujetos que participan del intercambio, la reformulación resulta dificultosa, como lo expresa el comentario eso no lo entendí (con la estructura elemento anafórico + elemento metadiscursivo/metacognitivo) y los intentos sucesivos de reformulación. El carácter figurado de la frase se reconoce como tal en la evaluación metadiscursiva sería una metáfora (con la estructura repetición de la expresión de referencia y elemento evaluativo metalingüístico), para finalmente llegar a una reformulación exitosa en la línea 19, que presenta, a su vez, un comentario metadiscursivo que indica cierta duda o distanciamiento: podemos decir. En consonancia, se registran atenuadores a lo largo del intercambio. Así, al contrario de lo que se podría suponer por su grado de lexicalización, la frase mojar la oreja es tratada por los hablantes que participan del intercambio como una expresión con un bajo grado de convencionalidad, que recibe una demanda explícita de reformulación, la cual, además, conlleva un esfuerzo evidente. Esto puede explicarse por las características socioetarias de los sujetos participantes.

Un ejemplo inverso es el que se transcribe a continuación (8), en el que la expresión metafórica churrito es fácilmente comprendida y reformulada, a pesar de que podría pensarse que su grado de convencionalidad es menor (ya que no está ligada a una metáfora conceptual y no se registra en diccionarios de lengua general, aunque sí en Conde 2011):

13 'Insultar o molestar a alguien para discutir con él'. 
(8) Yo tengo una amiga zarpada en atrevida; / cuando se va el novio, ya arranca pa' la esquina, / se prende un churrito y empieza a flashear / y con los pibitos la empezamos a enfiestar.

1 B bueno, cuando se:/ después dice se despierta el chorrito y le empieza/ y la empieza a flashear..

\begin{tabular}{rrl}
\hline 2 & C & no pará, \\
\hline 3 & C & se prende un churrito' para mí es que se prende \\
& & un cigarrillo, \\
\hline 4 & M & ay: es obvio que sí \\
5 & C & un porro \\
6 & D & y flashear' \\
\hline 7 & C & y sí, \\
8 & B & y sí, digamos', no creo que sea un cigarrillo, \\
\hline 9 & C & un cigarrillo no creo \\
\hline 10 & D & por qué no' \\
\hline 11 & B & porque un cigarrillo no te puede hacer nada \\
\hline 12 & D & sí pero, e:h le p/ lo que le po:nen esas co:sas,. sí, \\
\hline 13 & B & bueno un POrro,. droga, eso. toda=esas cosas \\
14 & D & sí \\
\hline 15 & D & no quería decirla la palabra pero la dijiste Bibi. \\
& & bueno, ya está, \\
\hline 16 & C & y qué querés <risas > \\
\hline 17 & D & y bueno, esa es la palabra;. \\
18 & C & y si es así
\end{tabular}

En el ejemplo anterior, el intercambio gira en torno a la adecuación de identificar churrito con droga. Pero esta duda respecto de la expresión metafórica no es lingüística en términos restringidos (el es obvio, así como el tono general del intercambio dejan claro que todos los participantes reconocen el sentido metafórico de churrito), sino comunicativa, tal como se refleja en el comentario metadiscursivo no quería decir la palabra: lo que se está sometiendo a evaluación no es el significado de la expresión, sino su adecuación en registro. La reformulación propuesta por C en las líneas 3 y 5, primero como cigarrillo e inmediatamente como porro, es rápidamente aceptada e incluso evaluada como obvia (línea 4). Esta evaluación metadiscursiva permite reconocer que, para los participantes, el uso de churrito como cigarrillo de marihuana se acerca más a la «norma»-es decir, que se usa con frecuencia (Hanks 2004, 2013)- de lo que los diccionarios de uso reflejan. Los intercambios posteriores giran en torno a la adecuación de 
las palabras droga y porro para el contexto comunicativo: los interlocutores se encuentran en la escuela y saben que su intercambio está siendo grabado. En efecto, cuando los hablantes evalúan sus enunciados lo hacen siguiendo una norma implícita, que puede ser de contenido o de uso (Gülich y Kotschi 1995). Así, puede apreciarse la tensión entre «no querer decir la palabra» (por considerarla inadecuada a la situación comunicativa) con la adecuación de la palabra al objeto (esa es la palabra). Los comentarios metadiscursivos, entonces, dan cuenta de esta tensión.

\subsection{Grado 3: Metáforas nuevas}

En este conjunto incluyo aquellas metáforas creadas más o menos ad hoc para un texto particular. Se trata de metáforas nuevas, creativas, dinámicas, que, como tales, no están convencionalizadas y se encuentran característicamente extendidas a lo largo del texto que las contiene (Hanks 2006). Dado que la reformulación de este tipo de metáforas es más trabajosa para los hablantes, quienes, por su bajo grado de convencionalidad, no tienen almacenada ninguna «norma» que les facilite su comprensión, era esperable que el trabajo metadiscursivo fuera importante, con gran presencia de calificadores. Sin embargo, en el corpus analizado esto no fue así. En los siguientes ejemplos (9 y 10) puede observarse que el trabajo metadiscursivo y la presencia de calificadores no son tan marcados, especialmente en comparación con el trabajo realizado para reformular expresiones metafóricas con un grado intermedio de convencionalidad (ejemplos 7 y 8). Dadas la dificultad que supone el trabajo reformulativo de este tipo de metáforas y la necesidad de realizar aproximaciones sucesivas, se encuentran diferentes atenuadores, que permiten dar cuenta del carácter aproximado de las reformulaciones que se van proponiendo.

(9) Pero, (porque) soy un albañil / que está reconstruyendo su vida,/ ya no soy más feliz./ Con cemento curo mis heridas, oh. / (Y) hoy te pido, como ayer, / que no me dejes caer. / Por favor, ¡dejame ser / aunque sea sólo un ladrillo en tu pared!

1 L después un ara/un arañil que está reconstruyendo su vida o sea de:

\begin{tabular}{lll}
\hline 2 & A & \&una metáfora \\
\hline 3 & L & sí una: eh tipo una metáfora e:h porque: no es lo que habla \\
4 & & de él. de: las cosas. de que por culpa de ella:: se sentía mal y \\
5 & & tienen que volver \\
\hline 6 & D & Indirectas \\
\hline
\end{tabular}


7 L a reconstruir su vida de vuelta,..

\begin{tabular}{rrl}
\hline 8 & L & Después \\
\hline 9 & A & cómo es \\
\hline 10 & L & con cemento curo mis heridas bue eso no sé,.. <risas $>$ \\
\hline 11 & A & como que puede ser trabajando.. trabajando \\
\hline 12 & L & que trabajando se olvida de las cosas' \\
\hline 13 & A & cla:ro eso puede ayudar a. a olvidar
\end{tabular}

En este ejemplo (9) puede observarse la presencia de comentarios que reconocen de manera metalingüística la expresión metafórica como tal ( una metáfora, un tipo de metáfora). Este fenómeno se registra en la reformulación de metáforas de diferente grado de convencionalidad: los sujetos reconocen que se encuentran ante la tarea de reformular una expresión metafórica y construyen la reformulación a partir de eso. En la reformulación de metáforas creativas, la colaboración entre los interlocutores se intensifica. Esto puede verse en la manera en que diferentes hablantes completan las frases iniciadas por sus interlocutores (encabalgamiento en la línea 2) y repiten expresiones formuladas por otros para construir, a partir de ahí, la reformulación (líneas 2 y 3 y 11 y 12).

(10) Estoy urgente, estoy conveniente, / sería bueno llamarte. / A pesar del miedo, / a pesar de esta cruz, / a pesar de esta mueca.

1 M bueno estoy urgente

2 D estoy urgente

3 B estoy urgente estoy conveniente llamarte, o sea'.

4 B que está:

5 M estoy urgente que estoy

6 B e:h cómo se dice'

7 M \&está apresurado, como si yo dijera

8 B no:

\begin{tabular}{rrl}
\hline 9 & B & estoy urgente, está:.. arrepenTI:do no ves'. estoy conveniente \\
10 & & sería bueno llamarte, \\
\hline 11 & M & algo de que está arrepentido \\
12 & D & estoy urgente estoy alerta \\
\hline 13 & B & por eso \\
\hline 14 & D & Claro \\
\hline 15 & M & sí está bien,
\end{tabular}

15 M sí está bien, 
En 10 también puede observarse el fenómeno de la repetición de las palabras de otro para construir la reformulación (líneas 9 y 11). En este ejemplo se realizan tres propuestas de reformulación para estoy urgente; los participantes se ven ante la necesidad de negociar y llegar a un acuerdo al respecto. En 7, M propone apresurado, comentando con como si yo dijera, que da cuenta de su falta de seguridad respecto de la reformulación propuesta. B expresa su desacuerdo (línea 8) y propone una reformulación alternativa de urgente como arrepentido, fundamentada en el cotexto posterior, que cita dirigiéndose específicamente a M (con el «no ves»). M expresa su acuerdo en 11, con la repetición de la propuesta de B. En cuanto a $\mathrm{D}$, realiza su reformulación al mismo tiempo que $\mathrm{M}$, de manera solapada (línea 12), con lo que es posible que no haya sido escuchada por los otros participantes o que se la haya considerado como equivalente, en tanto en las líneas finales todos expresan su acuerdo con la reformulación realizada. En este ejemplo, los comentarios metadiscursivos (cómo se dice y como si yo dijera) que se encuentran funcionan, asimismo, como atenuadores, dado que marcan el grado de inseguridad de los hablantes respecto de sus enunciados. No se producen evaluaciones ni se ahonda metadiscursivamente en la expresión por reformular.

\section{Conclusiones}

El análisis realizado permite arribar a algunas conclusiones. En primer término, puede afirmarse que los hablantes adolescentes hacen uso de procedimientos de calificación metadiscursiva cuando reformulan metáforas, como pudo observarse en los ejemplos. En muchas ocasiones, los sujetos que participaron de los intercambios muestran ser conscientes de que están reformulando una expresión metafórica, puesto que señalan su metaforicidad de manera explícita mediante diferentes comentarios y evaluaciones que son, lógicamente, metalingüísticos. Esto se registra en la reformulación de metáforas de todos los grados de convencionalidad, aunque es más frecuente en el grado intermedio.

En segundo lugar, el análisis de las huellas de calificación metadiscursiva en el marco de la reformulación de metáforas permitió identificar una correlación entre el grado de convencionalidad de la metáfora y el conjunto de recursos de calificación empleados por los hablantes. En efecto, ha podido observarse que el tipo y la frecuencia de los recursos lingüísticos para realizar los procedimientos de calificación varía, en este corpus, de acuerdo con el grado de convencionalidad de la expresión metafórica reformulada, pudiéndose establecer ciertas tendencias que se sistematizan en las siguientes correlaciones: 
1) En el conjunto de reformulaciones cuya ER presenta una metáfora con un alto grado de convencionalidad, el trabajo de formulación tiende a ser acotado y son nulos o escasos los comentarios o evaluaciones metadiscursivos.

2) En el conjunto de reformulaciones cuya ER presenta una metáfora con un grado de convencionalidad intermedio se registra la mayor variedad y profusión de huellas de calificación metadiscursiva. Las evaluaciones que aparecen con mayor frecuencia señalan apreciaciones respecto de la adecuación en registro de algún aspecto (léxico, sobre todo) de la interacción que se está llevando a cabo. Los comentarios, por su parte, no sólo aparecen para señalar la dificultad, sino que también, en ocasiones, indican la facilidad en la reformulación.

3) En el conjunto de reformulaciones cuya ER es una metáfora nueva no se registraron calificadores metadiscursivos en abundancia, aunque sí es frecuente la atenuación. La necesidad de colaboración en la realización de la reformulación se vuelve evidente para este conjunto: se aprecia la negociación y la construcción conjunta de la reformulación. Los intercambios son más fluidos y discontinuos; en ocasiones, los hablantes repiten palabras de otros o completan sus enunciados.

En tercer lugar, respecto de los criterios para determinar el grado de convencionalidad de una expresión metafórica y su carácter figurado, el análisis realizado mostró que, muchas veces, las huellas de calificación metadiscursiva pueden funcionar como indicadores lingüísticos respecto de hasta qué grado los sujetos perciben una metáfora como tal. Lo que constituye la «norma» de uso (Hanks 2004, 2006) de una expresión lingüística puede variar para diferentes grupos socioetarios, en tanto varían sus patrones de comportamientos lingüísticos: lo que es frecuente en el habla de ciertos grupos puede no serlo en la de otros. El grado de convencionalidad de una expresión metafórica debe ser considerado, en consecuencia, gradual y variable para diferentes grupos socioetarios y, por lo tanto, los criterios para establecer esa gradación deben ser múltiples. En este marco, los comentarios metadiscursivos pueden funcionar en el análisis como un parámetro adicional.

Por último, cabe destacar la necesidad de comprobar la validez de las conclusiones aquí expresadas en estudios posteriores más amplios respecto a la cantidad de sujetos involucrados, el grupo socioetario y la situación comunicativa en la que se producen los intercambios. 


\section{BIBLIOGRAFÍA}

ANTos, Gerard (1982): Grundlagen einer Theorie des Formulierens, Tübingen: Niemeyer.

BACH, Carme (2002): Els connectors reformulatius Catalans: Anàlisi i proposta d'aplicació lexicogràfica, Barcelona: UPF. <http://hdl.handle.net/10803/7487>.

Battaner, Paz (dir.) (2003): Diccionario de uso del español de América y España (DUAE) [CDRom], Barcelona: SPES.

BLAKEMORE, Diane (1993): «The relevance of reformulations», Language et Literatur $2 / 2,101-120$.

- (2002): Relevance and Linguistic Meaning. The semantics and pragmatics of discourse markers, Cambridge: Cambridge University Press.

BREgANT, Lucía (2015): «Las metáforas en los procedimientos de reformulación textual», ponencia presentada en el XI Congreso de ALED, Buenos Aires.

BRIZ, Antonio (1998): El español coloquial en la conversación: esbozo de pragmagramática, Barcelona: Ariel.

- et al. (1995): La conversación coloquial. Materiales para su estudio. Valencia: Universitat de València.

CAMERON, Lynne (2003): Metaphor in educational discourse, Londres: Continuum.

- y Alice Deignan (2003): «Combining large and small corpora to investigate tuning devices around metaphor in spoken discourse», Metaphor and Symbol $18 / 3,149-160$.

CiAPUSCIO, Guiomar (2005): «Las metáforas en la creación y recontextualización de la ciencia», Signo y Seña 14, 183-211.

- (2007a): «Acciones de calificación en conferencias de divulgación científica», Revista internacional de lingüistica iberoamericana 5/1, 59-75.

- (2007b): «Comentarios y evaluaciones del léxico en el discurso del científico: aspectos estructurales y funcionales». En Patricia Vallejos (coord.), Los estudios del discurso. Nuevos aportes de la investigación en Argentina, Bahía Blanca: Universidad Nacional del Sur, 37-58.

- (2016): «Conceptualizaciones metafóricas y recursos de formulación en narraciones de pacientes con migraña», Oralia 19, 39-60.

Conde, Óscar (2011): Diccionario etimológico del lunfardo, Buenos Aires: Taurus.

CzYŻEwski, Marek, et al. (1995): Selbst-und Fremdbilder im Gespräch. Theoretische und methodologische Aspekte. Nationale Selbst- und Fremdbilder im Gespräch, Opladen: Springer VS.

DeIGnAN, Alice (2008): «Corpus Linguistics and Metaphor». En Raymond Gibbs Jr. (ed.), The Cambridge Handbook of Metaphor and Thought, Nueva York: Cambridge University Press, 280-294.

DuARTE, Mercedes (2016): «Redes de metáforas cognitivas en el discurso político: "el socialismo del siglo XxI" de Hugo Chávez», Revista latinoamericana de estudios del discurso 13/1, 57-78. 
Fernández Colomer, María José (2003): «La metáfora en el español coloquial», Revista Interlingüistica 16, 359-373.

Figueras, Carolina (2001): «Reflexiones en torno a las estrategias de reformulación parafrástica en la oralidad y en la escritura». En María Antonia Martín Zorraquino (ed.), ¿Qué español enseñar?: norma y variación lingüisticas en la enseñanza del español a extranjeros, Zaragoza: ASELE/Universidad de Zaragoza, 297-309.

Fuentes Rodríguez, Catalina (1993): «Conclusivos y reformulativos», Verba 20, 171-196.

Garcés Gómez, María del Pilar (2008): La organización del discurso: marcadores de ordenación y reformulación, Madrid: Iberoamericana.

GüLICH, Elisabeth (2006): «Des marqueurs de structuration de la conversation aux activités conversationnelles de structuration: Réflexions méthodologiques». En Martina Drescher (ed.), Les marqueurs discursifs dans les langues romanes, Frankfurt: Peter Lang, 11-36.

- y Thomas Kотsсні (1987): «Les actes de reformulation dans la consultation 'La dame de Caluire'». En Pierre Bange (ed.), L'analyse des interactions verbales. La Dame de Caluire: une consultation, Berna: Peter Lang, 15-81.

- y - (1995): «Discourse production in oral communication. A Study Based on French». En Uta Quasthoff (ed.), Aspects of oral communication, Berlín: Mouton de Gruyter, 30-66.

HANKs, Patrick (2004): «The Syntagmatics of Metaphor», International Journal of Lexicography 17/3, 245-274.

- (2006): «Metaphoricity is a gradable». En Anatol Stefanowitsch y Stefan Gries (eds.), Corpora in Cognitive Linguistics, Berlín/Nueva York: Mouton de Gruyter, I, $17-35$.

- (2013): Norms and Exploitations, Cambridge: MIT Press.

HERnÁNDEZ Miranda, Natalí (2015): «La construcción mediática de perdedores y vencedores en el campo político. Análisis de las metáforas, tópicos y lexicalización», Cuadernos de Lingüistica Hispánica 25, 81-98.

Koтschi, Thomas (1986): «Procédés d'évaluation et de commentaire métadiscursifs comme stratégies interactives», Cahiers de Linguistique Française 7, 207-230.

Kövecses, Zoltan (2002): Metaphor: A Practical Introduction, Nueva York: Oxford University Press.

LAKOFF, George (1993): «The contemporary theory of metaphor». En Andrew Ortony (ed.), Metaphor and thought, Cambridge: Cambridge University Press, 202-251.

—, Jane Espenson y Alan Schwartz (1991): Master metaphor list. Berkeley, Cognitive Linguistics Group. <http://araw.mede.uic.edu/ alansz/metaphor/ METAPHORLIST.pdf $>$.

— y Mark Johnson (1980): Metaphors We Live By, Chicago: University of Chicago Press.

— y - (1999): Philosophy in the Flesh, Nueva York: Basic Books.

Olza Moreno, Inés (2008): «Metáfora y argumentación en el lenguaje político y la prensa españoles: aproximación a las metáforas relacionadas con el "proceso de paz"», Estudios de Lingüística 22, 213-242. 
Plager, Federico (coord.) (2008): Diccionario integral del español de la Argentina (DIEA). Buenos Aires: Tinta Fresca/Voz Activa.

Pons Bordería, Salvador (2013): «Un solo tipo de reformulación», Cuadernos AISPI 1/2, 151-171.

REAl ACAdEMIa EsPañola (2001): Diccionario de la lengua española (DRAE) (23. ${ }^{a}$ ed.) $<$ http://dle.rae.es/?w=diccionario $>$.

RiBAs, Montserrat y Júlia TODOLí (2008): «La metáfora de la mujer objeto y su reiteración en la publicidad», Discurso \& Sociedad 2/1, 153-169.

Rossari, Corinne (1994): Les opérations de reformulation, Berna: Peter Lang.

Roulet, Eddy (1987): «Completude interactive et connecteurs reformulatifs», Cahiers de Linguistique Française 8, 111-140.

SCHEGLOFF, Emanuel, Gail JEFFERSON y Harvey SACKS (1977): «The preference for self-correction in the organization of repair in conversation", Language 53/2, 361-382.

SORIANO, Cristina (2012): «La metáfora conceptual». En Iraide Ibarretxe-Antuñano y Javier Valenzuela (coords.), Lingüistica cognitiva, Barcelona: Anthropos, 97-121.

STEEN, Gerard (2007): Finding metaphor in grammar and usage. Ámsterdam / Filadelfia: John Benjamins. 


\section{APÉNDICE}

\section{CONVENCIONES DE TRANSCRIPCIÓN}

Utilizo una versión adaptada del método de transcripción del grupo de análisis de la conversación de la Universidad de Bielefeld (Czyżewski et al. 1995). Lo que se expresa de manera simultánea, en lo posible, se escribe directamente debajo. Las líneas horizontales tienen la función de facilitar la visualización de las intervenciones simultáneas (a la manera de una partitura musical). Los caracteres que se utilizan en un significado distinto de la ortografía estándar se listan a continuación:

\begin{tabular}{ll}
\hline \multicolumn{1}{l}{} & Curva entonacional ascendente \\
\hline, & Curva entonacional descendente \\
\hline MAYÚSCULAS & Énfasis \\
\hline$:$ & Alargamiento \\
\hline$/$ & Interrupción o corrección \\
\hline$\cdot \quad .$. & Pausa: muy corta, corta, larga \\
\hline$\&$ & Encabalgamiento rápido de intervenciones \\
\hline$<$ Comentario $>$ & \\
\hline
\end{tabular}

